\title{
Regional Variation of Distal Esophagus Distensibility Assessed Using the Functional Luminal Imaging Probe (FLIP)
}

\author{
Zhiyue Lin, Frédéric Nicodème, Lubomyr Boris, Chen-Yuan Lin, Peter J Kahrilas, and John \\ E Pandolfino \\ Department of Medicine, Feinberg School of Medicine, Northwestern University, Chicago, IL, \\ USA
}

\begin{abstract}
Background-This study aimed to evaluate the spatial variation of esophageal distensibility in normal subjects using a novel multichannel functional luminal imaging probe (FLIP).

Methods-Ten healthy subjects (4 male, age 21-49 years) were evaluated during endoscopy with a high-resolution impedance planimetry probe (FLIP) positioned through the esophagogastric junction (EGJ) and distal $10 \mathrm{~cm}$ of the esophageal body. Stepwise bag distensions using $5 \mathrm{ml}$ increments from 0 to $60 \mathrm{~mL}$ were conducted and simultaneous measurements of cross-sectional area (CSA) and the associated intrabag pressure from each subject were analyzed using a customized MATLAB ${ }^{\mathrm{TM}}$ program. The distensibility along the esophagus was determined and compared between the EGJ and interval locations at $2-5 \mathrm{~cm}$ and $6-10 \mathrm{~cm}$ above the EGJ.
\end{abstract}

Results-The pressure-CSA relationship was nearly linear among sites at lower pressures ( 0 to $7.5 \mathrm{mmHg}$ ) and reached a distension plateau at pressures ranging from 8 to $24 \mathrm{mmHg}$. The location of greatest distensibility was $4 \mathrm{~cm}$ above the EGJ. Although the CSAs of individual recording loci were not significantly different, there was a significant difference between the mean CSAs when comparing the region 2 to $5 \mathrm{~cm}$ proximal to EGJ to that 6 to $10 \mathrm{~cm}$ proximal to the EGJ.

Conclusions-There were significant regional differences in distensibility along the distal esophagus with lower values in the proximal part compared to more distal part. The greatest distensibility was noted to occur at about $4 \mathrm{~cm}$ above the EGJ in close proximity to the location of the contractile deceleration point and phrenic ampulla.

\section{Keywords}

cross-sectional area; esophageal distensibility; functional imaging; impedance planimetry

Correspondence: John E. Pandolfino, MD, MSCI, Northwestern University, Feinberg School of Medicine, Department of Medicine, 676 St Clair St, Suite 1400, Chicago, Illinois 60611-2951, Tel:312-695-4729, Fax: 312-695-3999, j-pandolfino@northwestern.edu.

\section{AUTHOR CONTRIBUTIONS}

Zhiyue Lin: Analysis and interpretation of data, drafting of the manuscript, approval of the final version

Frédéric Nicodème: Analysis and interpretation of data, approval of the final version

Lubomyr Boris and Chen-Yuan Lin: Acquisition and analysis of data, approval of the final version

Peter J. Kahrilas: Study concept and design, revising of the manuscript critically, approval of the final version

John E. Pandolfino: Study concept and design, analysis and interpretation of data, revising the manuscript critically, approval of the

final version

COMPETING INTERESTS

John E. Pandolfino [Given imaging (consulting, educational)]

No other conflicts for remaining authors (ZL, FN, LB, CL, PJK) 


\section{INTRODUCTION}

Although the assessment of esophageal transport has primarily focused on describing the vigor and propagation velocity of the peristaltic wavefront, the function of esophageal emptying has other important components that are not appreciated with manometric techniques. While peristalsis is important in generating a sufficient intrabolus pressure for trans-EGJ flow, emptying can occur without peristalsis and thus, gravity, mechanical properties of the esophageal wall, tonic contractions in both the circular and longitudinal muscle and EGJ opening dimensions are also important in generating a sufficient intrabolus pressure in the esophagus to drive esophageal emptying.

Even though manometry can provide surrogate information regarding the effect of mechanical and tonic contractile properties of the esophageal wall on bolus emptying dynamics, it cannot display the individual contributions of these components of esophageal wall distensibility and opening dimensions of the EGJ. This is an important limitation of our current armamentarium to study and treat disorders of the esophagus as alterations in the response of the esophagus to a distensile force may be important for determining symptoms and bolus transit. Disorders associated with reduced compliance, such as eosinophilic esophagitis (EoE), will cause obstruction and increased wall strain with normal bolus volumes while disorders associated with increased compliance, such as advanced achalasia, will reduce the ability of the esophagus to develop adequate emptying pressures. Thus, it is logical that measurement of mechanical properties of the esophageal wall and response to distention should be included in our current assessment of esophageal function.

Currently, impedance planimetry techniques appear to be the most promising method to study how the esophagus responds to distention as this provides a measure of the relationship of CSA and pressure during volume distention (1-8). However, a major challenge with this technique is that esophageal compliance and tonic activity may not be uniform along the esophagus $(2,4)$ and measurement with a single sensor impedance planimetry probe or short balloon are associated with movement artifact. Although multichannel high resolution impedance measurements can now be obtained by using the functional luminal imaging probe (EndoFLIP, Crospon Medical Devices, Galway, Ireland), this technique has only been applied to study the narrowest luminal cross-sectional area (CSA) and corresponding pressure at the EGJ $(1,5,6)$ or the esophageal body in EoE $(3,7,8)$. Although the length of the FLIP is longer than standard impedance planimetry, it is still limited by movement and the lack of a standardized reference for accurate location.

In order to study the region-specific variation of distensibility along the esophagus, we have developed a technique using sophisticated filtering methods coupled with a topographic data presentation format that displays changes in esophageal diameter as a continuum along the length of the esophagus (7). We hypothesize that this technique will provide a more accurate description of potential regional differences in the interaction between the mechanical properties of the esophageal wall and tonic activity of the esophageal wall musculature as differences in distensibility can be accurately mapped using the EGJ as a reference point. Thus, the aim of this study was to assess the regional variation of esophageal distensibility in the distal esophagus and through the EGJ in normal subjects using these novel modifications in FLIP measurement and analysis.

\section{METHODS}

\section{Subjects}

Ten asymptomatic healthy volunteers ( 4 male, age 21-49 years) were studied. All subjects underwent upper endoscopy to evaluate for hiatus hernia, esophagitis, stricture, or mucosal 
changes suggestive of EoE. None of the subjects had a history of gastrointestinal surgery or malignancy. The study protocol was approved by the Northwestern University Institutional Review Board and informed consent was obtained from each subject.

\section{Modified EndoFLIPTM system and study protocol}

The EndoFLIP assembly (Crospon Inc., Galway, Ireland) used was mounted on a $240 \mathrm{~cm}$ long, $3 \mathrm{~mm}$ outer diameter catheter. An infinitely compliant bag (up to a volume of $60 \mathrm{ml}$ ) mounted on the distal $18 \mathrm{~cm}$ of the probe was fabricated to assume a $16 \mathrm{~cm}$ long cylindrical shape with tapering ends. The $16 \mathrm{~cm}$ impedance planimetry segment within the bag was comprised of 17 ring electrodes spaced one $\mathrm{cm}$ apart. The minimal-to-maximal range of CSA measurable within the infinitely compliant range was $21-380 \mathrm{~mm}^{2}$ over the infinitely compliant range. Values above $380 \mathrm{~mm}^{2}$ could be measured, however, this would occur in the distention range at which the mechanical properties of the bag would be engaged. The assembly also contained a solid-state pressure transducer for determining intrabag pressure. In order to avoid an overestimation of pressure by a contraction localized to the site of the pressure sensor, we have ignored all pressure data when the impedance planimetry measurement suggested that the bag was closed or collapsed at the pressure sensor site in the distal end of the bag. Measurements from the 16 impedance planimetry electrode pairs and the pressure transducer were sampled at $10 \mathrm{~Hz}$ with the data acquisition system and transmitted to the recording unit. A detailed description of the concepts and components of the EndoFLIP system has been previously published $(1,5)$.

Subjects underwent endoscopy in the left lateral decubitus position to help position the FLIP for distensibility measurements. Moderate sedation with 5-10 mg midazolam and 0-200 $\mu \mathrm{g}$ fentanyl was administered during the procedure. The EndoFLIP probe was placed transorally during endoscopy and the distal end of the probe was positioned in the proximal stomach. The endoscope was withdrawn before the study was started. Cross-sectional areas within the esophagus were measured during $5 \mathrm{ml}$ stepwise distensions beginning with $5 \mathrm{ml}$ and increasing to a maximum of $60 \mathrm{ml}$ (each step lasted for 5-20 s). To prevent unintended dilation of a stiff or poorly compliant esophagus, the recording unit was set to stop infusing and display an alarm message if the intrabag pressure exceeded $60 \mathrm{mmHg}$.

\section{Data Analysis}

Distension volume, intrabag pressure, and 16 channels of impedance planimetry data from each subject were exported to MATLAB (The Math Works, Natick, MA) for further analysis using a customized MATLAB program (Figure 1). This program: 1) applied a median filter to intrabag pressure and CSA data to minimize vascular and respiratory artifact, 2) identified the midline of the EGJ by searching for the minimal CSA values on the distal 6 channels and reconfigured the data array from this landmark, 3) extracted the maximal CSA values for each channel at $2 \mathrm{mmHg}$ pressure increments to establish the CSApressure relationship, and 4) generated a plot of maximal CSA data changes with the corresponding pressure values along with a 'FLIP topography' plot that described regional variation relative to the EGJ of CSA vs pressure (7) (Figure 1). Use of the narrowest segment localized at the diaphragmatic hiatus as a marker for the EGJ in non-hernia subjects has been validated using barostat (9) and hydrostat (10) techniques. In addition, the median values of intrabag pressure and narrowest CSA data during each distension period for the EGJ (distal 4-6 channels) and the esophageal body $2 \mathrm{~cm}$ above the EGJ midline were derived. (7) The EGJ and esophageal body distensibility index at each distension volume was calculated as narrowest CSA of each region in $\mathrm{mm}^{2} / \mathrm{mmHg}(1,3,7)$. 


\section{Statistical Analysis}

Results were expressed as median (interquartile range (IQR) or $5^{\text {th }}-95^{\text {th }}$ percentile). The differences in distensibility data among esophageal axial levels and between the EGJ and the esophageal body were analyzed and compared using the Wilcoxon rank sum test for paired testing and the Kruskal Wallis test with more than 2 groups with a significance level set at $\mathrm{P}<0.05$.

\section{RESULTS}

Median CSAs at 7.5, 15, and 30-mmHg intrabag pressures from the EGJ and distal esophagus from 10 subjects are shown in the Table 1. The corresponding changes in median esophageal CSA with stepwise increments of intrabag pressure are shown in Figure 2 and as a FLIP topography plot in Figure 3. Evident in Figure 2, the pressure-CSA relationship was nearly linear among locations at lower pressures $(0-10 \mathrm{mmHg})$ and reached a distension plateau at pressures ranging from $10-30 \mathrm{mmHg}$. The focus of greatest CSA was in the distal esophagus $4 \mathrm{~cm}$ proximal to the midpoint of the EGJ (Figures 2 and 3). Although the CSAs of individual recording loci were not significantly different, there was a significant difference between the median CSAs comparing the region $2-5 \mathrm{~cm}$ proximal to EGJ to that $6-10 \mathrm{~cm}$ proximal to the EGJ (Figure 2).

Table 2 shows results of median distensibility index (DI, $\mathrm{mm}^{2} / \mathrm{mmHg}$ ) values for the EGJ, esophageal body $2-5 \mathrm{~cm}$ proximal to the EGJ and esophageal body $6-10 \mathrm{~cm}$ proximal to the EGJ at distension volumes 30, 40, 50 and $60 \mathrm{ml}$. Consistent with the CSA-pressure relationship evident in Figures 2 and 3, the DI values among these loci were significantly different at all distension volumes. Among the three, the region $2-5 \mathrm{~cm}$ proximal to EGJ had the greatest DI values. Moreover, when the distention volume increased from $30 \mathrm{ml}$ to 60 $\mathrm{ml}$; the DI values increased in the EGJ region but decreased in the region 2 to $5 \mathrm{~cm}$ proximal to EGJ and the region 6-10 $\mathrm{cm}$ above the EGJ suggesting that the limiting diameter of the bag was engaged at lower pressures in the esophageal body compared to the EGJ.

\section{DISCUSSION}

In this study, we used a novel multichannel intraluminal impedance planimetry probe to assess simultaneous measurements of 16 channels of CSA and corresponding intrabag pressure through the EGJ and smooth muscle segment of the esophageal body during volume distention in 10 healthy control subjects. Our findings support that there are significant regional differences in distensibility along the distal esophagus with the greatest distensibility noted to occur at about $4 \mathrm{~cm}$ above the EGJ. Additionally, the study suggests that opening of the esophageal body to diameters approximating $20 \mathrm{~mm}$ (60 French) occurs at pressures below $10 \mathrm{mmHg}$ along the entire length of the esophagus whereas the pressure required to open the EGJ to a similar dimension required a $30 \mathrm{mmHg}$ distention pressure. These findings are in line with expected physiologic manometric parameters associated with normal bolus transit through the esophagus (11) and provide a useful comparative reference to study various disease states.

Although manometry and fluoroscopy provide important information regarding pressure dynamics of bolus transit (11) and some data regarding opening diameters of the esophageal body and EGJ (12), the two techniques are unable to provide accurate estimates of the biomechanical and tonic contractile properties of the esophageal wall and the expected conformational changes that would occur when the esophagus encounters a distensile stress event. The FLIP probe was designed to measure CSA and pressure during a volume distention protocol similar to the barostat, but with the important advantage of providing anatomical information and an assessment of regional variations in distensibility. Given the 
fact that the esophagus is a reactive muscular organ, it is impossible to truly dissect out the effect of the passive material properties of the esophageal wall from the tonic contractile muscle forces that are engaged during esophageal distention. Therefore, the term distensibility as it relates to EndoFLIP analysis in the esophagus should be restricted to a measure of change in CSA in response to a pressure or volume distention. Thus, it should not be utilized interchangeably with elasticity or compliance.

Originally, this device was developed primarily to study the EGJ, however, we have modified this technology to measure the distensibility of the esophageal wall to assess food impaction risk in patients with eosinophilic esophagitis $(3,8)$. This assessment was focused on targeting the narrowest section of the esophagus to define risk for solid food dysphagia and food impaction as the disease process in EoE is focused on a change in the material properties of the esophageal wall. Our early results support that this technique could predict risk for food impaction, however, there was no landmark to confidently determine the location of the potential obstruction.

Given these limitations, the FLIP technique was further modified to generate a fixed landmark at the EGJ and extend measurements up the length of the esophagus to provide a more accurate depiction of the regional variability in distensibility. This modification entailed using a longer balloon and also developing a new data analysis paradigm to reference analysis to the EGJ. The midline of EGJ was defined by searching for the minimal CSA values on the distal 6 channels of CSA data, and reconfiguring the CSA data array from this landmark to $10 \mathrm{~cm}$ proximal (Figures 1 and 3). This data preprocessing step allowed us to extract the maximal CSA value at known intervals above the EGJ and provide a simultaneous assessment of EGJ distensibility. The data was further refined using filtering techniques to remove artifacts from vascular signals and respiratory fluctuations and was presented using FLIP topography plots so that a complete evaluation along the entire distention protocol could be assessed with relevant landmarks to assess the regional changes of distensibility along the esophagus.

Using these new modifications, our results suggest that the most distensible portion of the esophagus was approximately $4 \mathrm{~cm}$ above the EGJ. This was interesting as this location corresponds to the contractile deceleration point where the distal esophagus is noted to distend in a globular conformation during the final stages of emptying (13). A previous study by Patel et al in 11 healthy volunteers using single impedance planimetry measurements at 5,10 and $15 \mathrm{~cm}$ interval above the EGJ suggested that the most distensible portion of the esophageal body was localized to $10 \mathrm{~cm}$ above the EGJ and the least distensible section was localized in the proximal esophagus $5 \mathrm{~cm}$ below the UES (2). Although those results are slightly different from our results, we speculate that the prior study may have been limited by reduced resolution and movement artifact. Unfortunately, our study did not assess distensibility more proximally and a FLIP device that could extend above the transition zone would be helpful in further assessing the regional variation as the transition zone might have a unique mechanical profile.

Another interesting aspect of this analysis was that the FLIP topography analysis provided a unique perspective of the interaction between distensibility and peristalsis. Of note, the esophageal body opened at very low pressures consistent with the observation that gravity and tongue propulsion easily fill the esophagus in normal subjects. These opening diameters were obtained with minimal esophageal reactivity as evidence in panels A and C in Figure 1. Significant reactivity to distention did not occur until the distensibility plateau was reached and suggests that the threshold stimulus for distention-related contraction is linked to the distensibility plateau. Whether this is related to tension or stretch receptors is unclear and 
further research should include pharmacologic manipulation to alter the pressure-diameter relationship.

There were some limitations in the current study that could be improved by modifications in the device. Unfortunately, the FLIP device cannot distinguish the degree to which each esophageal wall layer is contributing to the distensibility measured during the distention protocol. Previous data support that the mucosa and submucosa are less compliant than the muscularis as evidenced by wider opening angles during the zero load state (14). However, the dominant contributor to wall distensibility may be different based on the underlying disease process and thus, FLIP analysis could be complemented by ultrasound imaging to highlight the interaction of distention and esophageal wall layers (15).

Our technique was also limited by the fact that the bag diameter was only $22 \mathrm{~mm}$ in diameter and distention beyond this range would incorporate elastic properties of the FLIP bag. This diameter was originally chosen based on fluoroscopic data obtained during our pilot work in which we focused on developing a tool that assessed distensibility along the working range of esophageal dilators. This range was also in line with esophageal diameter ranges presented by Lee et al (12) and thus, the distensibility values we have presented are relevant along the physiologic range of normal intrabolus pressure values during swallowing. Despite the fact that we observed distal esophageal distensibility values greater than $380 \mathrm{~mm}^{2}$ during volumetric distensions associated with intra-bag pressures greater than $20-30 \mathrm{mmHg}$, these data need to be interpreted with caution based on the limitations of the device. Future modifications to allow for a bag diameter of $30 \mathrm{~mm}$ would permit a more accurate assessment of the mechanical properties to a greater degree.

In summary, we have adapted the FLIP technique to assess simultaneous CSAs during volumetric distention through the EGJ and smooth muscle esophagus to provide a more comprehensive assessment of the response of the esophageal wall to distention in healthy control subjects. Our previous data suggest that this technique is useful in estimating risk of food impaction in EoE (8), however, this new approach could also be helpful in determining whether differences in distensibility can predict symptoms of dysphagia in patients with borderline motility disorders and seemingly normal endoscopic evaluations. Additionally, changes in distensibility related to muscle hypertrophy, submucosal/mucosal inflammation and fibrosis or tonic contraction of the muscle walls could also be explored as possible contributors of symptoms of chest pain and/or dysphagia in diseases where these defects are prominent. Future research should focus on using pharmacologic manipulation to study the varying effects of active and passive tone and incorporating complementary techniques, such as manometry and ultrasound.

\section{Acknowledgments}

\section{DISCLOSURES}

This work was supported by R01 DK079902 (JEP) and R01 DK56033 (PJK) from the Public Health Service.

\section{References}

1. Kwiatek MA, Pandolfino JE, Hirano I, et al. Esophagogastric junction distensibility assessed with an endoscopic functional luminal imaging probe (EndoFLIP). Gastrointest. Endosc. 2010; 72:272278. [PubMed: 20541755]

2. Patel RS, Rao SS. Biomechanical and sensory parameters of the human esophagus at four levels. Am. J. Physiol. 1998; 275:G187-G191. [PubMed: 9688644]

3. Kwiatek MA, Hirano I, Kahrilas PJ, et al. Mechanical properties of the esophagus in eosinophilic esophagitis. Gastroenterology. 2011; 140:82-90. [PubMed: 20858491] 
4. VINTER JENSEN L, Juhl CO, Gregersen H. Regional differences in passive elastic wall properties of the oesophagus: an impedance planimetric study in pigs. Neurogastroenterol Motil. 2008; 6:233238.

5. Mcmahon B-P, Frøkjær JB, Kunwald P, et al. The functional lumen imaging probe (FLIP) for evaluation of the esophagogastric junction. AJP: Gastrointestinal and Liver Physiology. 2006; 292:G377-G384.

6. Rohof WO, Hirsch DP, Kessing BF, et al. Efficacy of treatment for patients with achalasia depends on the distensibility of the esophagogastric junction. Gastroenterology. 2012; 143:328-335. [PubMed: 22562023]

7. Lin Z, Kahrilas PJ, Xiao Y, et al. Functional luminal imaging probe topography: an improved method for characterizing esophageal distensibility in eosinophilic esophagitis. Therap Adv Gastroenterol. 2013; 6:97-107.

8. Nicodème F, Hirano I, Chen J, et al. Esophageal Distensibility as a Measure of Disease Severity in Patients with Eosinophilic Esophagitis. Clin. Gastroenterol. Hepatol. 2013 Apr 13. Clin Gastroenterol Hepatol. doi:pii: S1542-3565(13)00459-X. 10.1016/j.cgh.2013.03.020. [Epub ahead of print].

9. Pandolfino JE, Shi G, Curry J, et al. Esophagogastric junction distensibility: a factor contributing to sphincter incompetence. Am. J. Physiol. Gastrointest. Liver Physiol. 2002; 282:G1052-G1058. [PubMed: 12016131]

10. Pandolfino JE, Shi G, Trueworthy B, et al. Esophagogastric junction opening during relaxation distinguishes nonhernia reflux patients, hernia patients, and normal subjects. Gastroenterology. 2003; 125:1018-1024. [PubMed: 14517784]

11. Ren J, Massey BT, Dodds WJ, et al. Determinants of intrabolus pressure during esophageal peristaltic bolus transport. [Internet]. Am. J. Physiol. 1993; 264:G407-G413. Available from: http://eutils.ncbi.nlm.nih.gov/entrez/eutils/elink.fcgi? dbfrom=pubmed \&id=8460696\&retmode=ref\&cmd=prlinks. [PubMed: 8460696]

12. Lee J, Huprich J, Kujath C, et al. Esophageal Diameter Is Decreased in Some Patients With Eosinophilic Esophagitis and Might Increase With Topical Corticosteroid Therapy. Clinical Gastroenterology and Hepatology. 2012; 10:481-486. [PubMed: 22309879]

13. Pandolfino JE, Leslie E, Luger D, et al. The contractile deceleration point: an important physiologic landmark on oesophageal pressure topography. Neurogastroenterol Motil. 2010; 22:395-400. [PubMed: 20047637]

14. Gregersen H, Lu X, Zhao J. Physiological growth is associated with esophageal morphometric and biomechanical changes in rats. Neurogastroenterol. Motil. 2004; 16:403-412. [PubMed: 15305995]

15. Abrahao L, Bhargava V, Babaei A, et al. Swallow induces a peristaltic wave of distension that marches in front of the peristaltic wave of contraction. Neurogastroenterol Motil. 2011; 23:201207. e110. [PubMed: 21083789] 

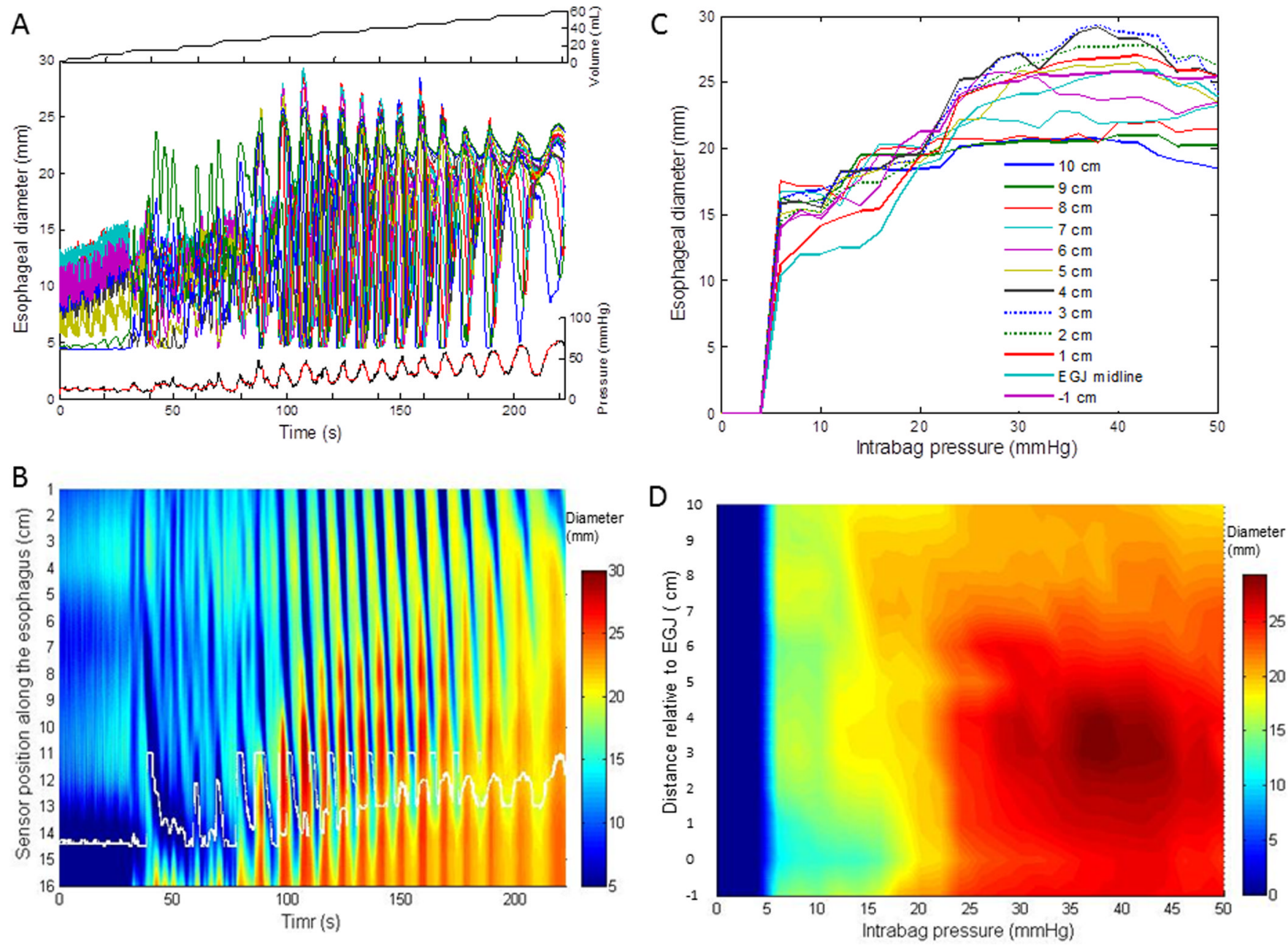

Figure 1.

Methodology for processing the FLIP data and quantifyzing the CSA-pressure relationship.

(A) The top panel shows the volumetric distention increments, the middle panel displays the 16 channels of raw diameter data and the lower panel the corresponding intrabag pressure data before (black line) and after median filtering (red line) from a control subject. (B) A FLIP topography plot assessing changes in diameter using a color scale is shown as a function of time during volume distention. Data are interpolated among the 16 channels. The white curves in the lower part of the FLIP topography plot represents the midline of the esophagigastric junction (EGJ). (C) The changes in 12 channels of esophageal diameters during stepwise increments in intrabag pressure at $-1 \mathrm{~cm}$ to EGJ midline (position 0 ) and at EGJ midline to $10 \mathrm{~cm}$ along the esophagus are graphed to illustrate the CSA-pressure relationship. (D) A new FLIP topography plot was generated after reconfiguration of the diameter data array using the midline of EGJ (position 0 ) as the reference and normalizing the data to focus on the $10 \mathrm{~cm}$ proximal to the EGJ. The diameter data is displayed as a function of pressure and time is not an input variable. 


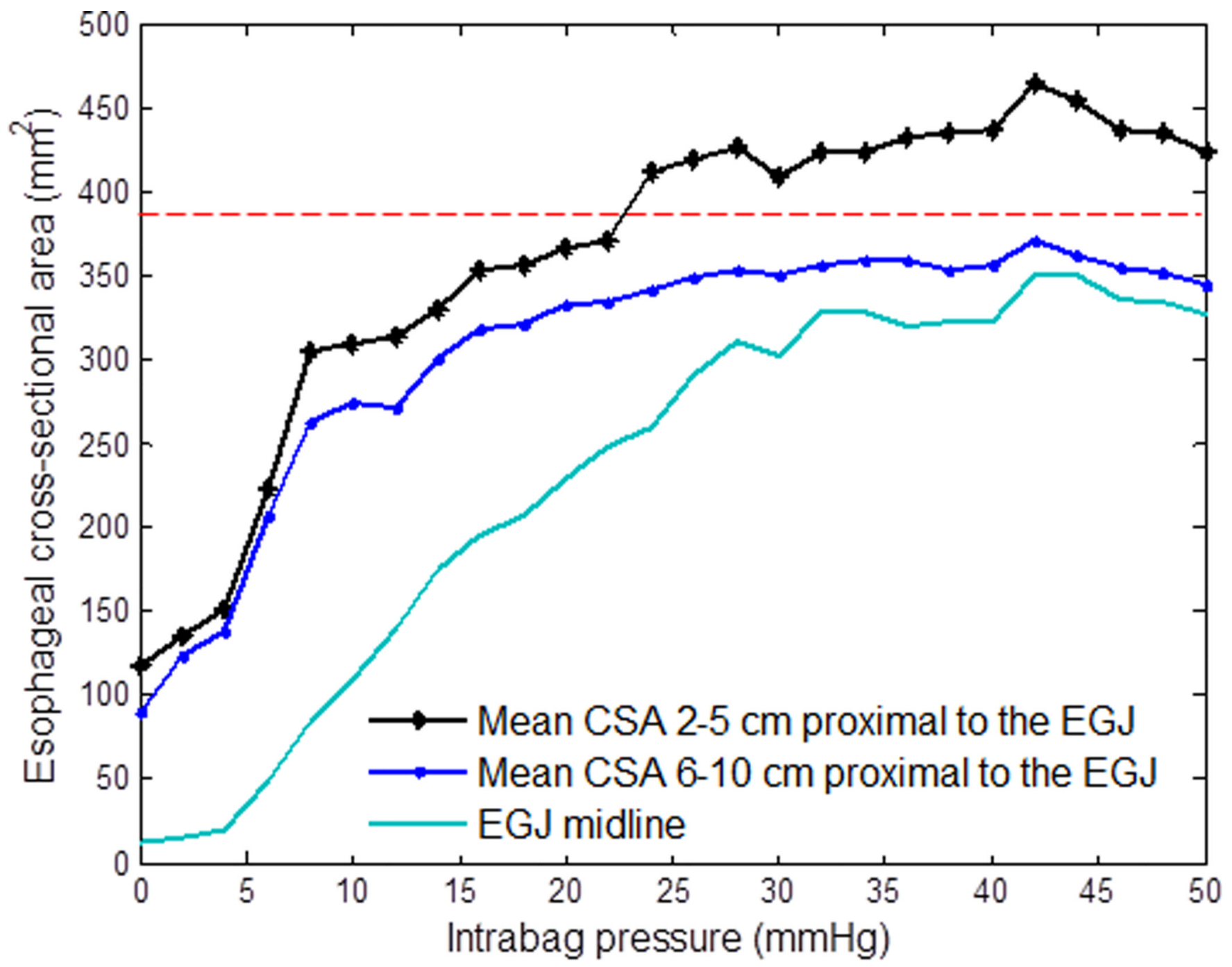

Figure 2.

Median CSA values at the EGJ midline, from 2 to $5 \mathrm{~cm}$ proximal to the EGJ, and from 6 to $10 \mathrm{~cm}$ proximal to the EGJ as a function of stepwise increments in intrabag pressure in 10 subjects. The red line depicts the maximal diameter of the bag where the intrinsic material properties of the bag are not engaged. Note, that the mean CSA 2 to $5 \mathrm{~cm}$ proximal to the EGJ reaches this limit at a pressure just above $20 \mathrm{mmHg}$ and the region from 6 to $10 \mathrm{~cm}$ proximal to the EGJ does not reach this limit throughout the study. 


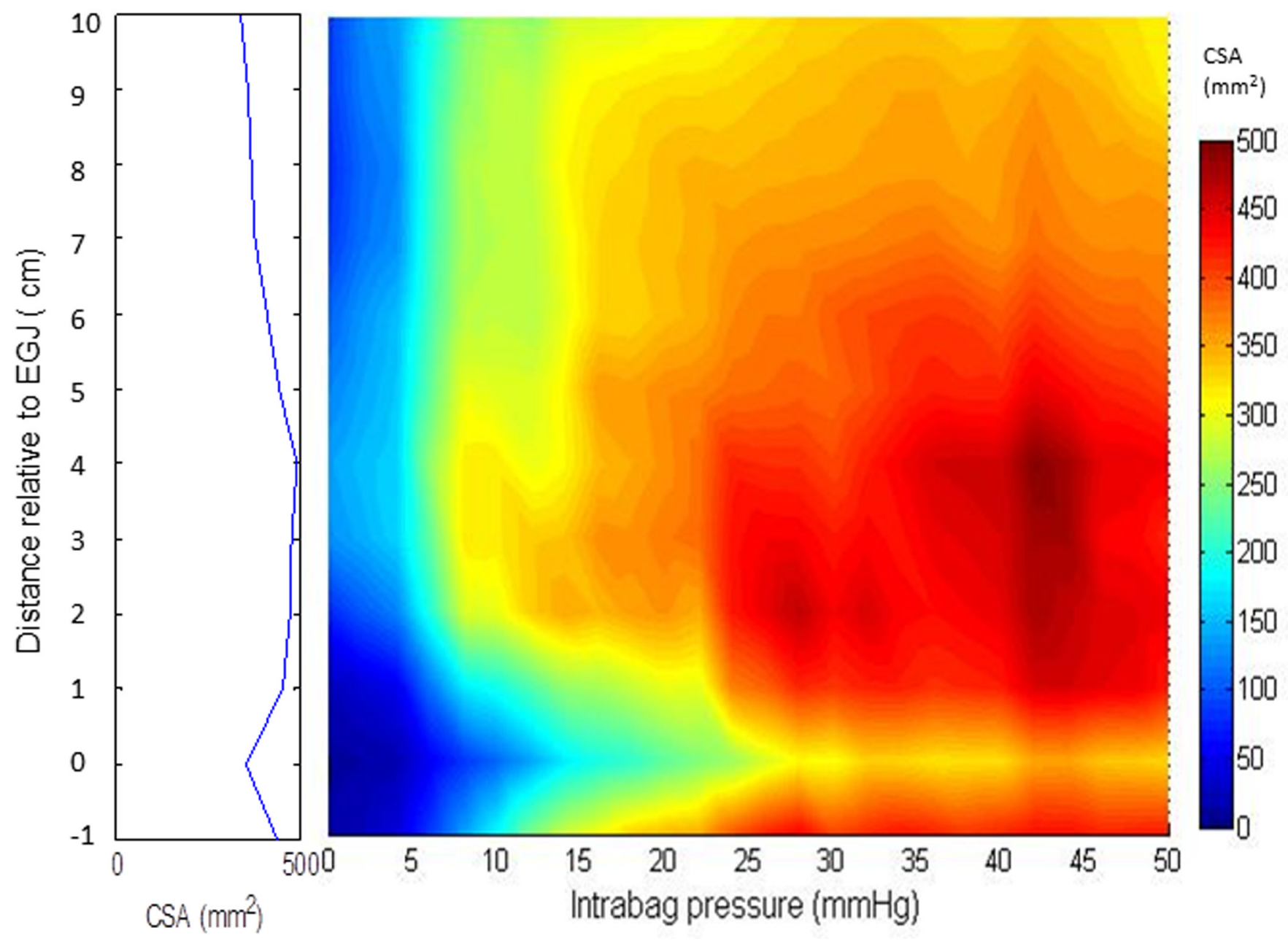

Figure 3.

A FLIP topography plot illustrates the median esophageal CSA values as a function of intrabag pressure using a color scale and interpolation among the 12 channels in 10 subjects, showing the regional variation of CSA vs pressure with distance relative to the EGJ (position 0). The maximal CSA values at each location are projected to the left as a spatial diameter variation plot. Note the increased maximal diameters are greatest in the range 2 to $4 \mathrm{~cm}$ above the EGJ (position 0). 
Table 1

Esophageal cross-sectional area (CSA) values at 7.5, 15 and $30 \mathrm{mmHg}$ intrabag pressure from the esophagogastric junction (EGJ) to $10 \mathrm{~cm}$ proximal in 10 subjects.

\begin{tabular}{|c|c|c|c|}
\hline & $\operatorname{CSA}\left(\mathbf{m m}^{2}\right)$ & & \\
\hline $\begin{array}{l}\text { Distance from the } \\
\text { midline of EGJ }(\mathbf{c m})\end{array}$ & $7.5 \mathrm{mmHg}$ & $15 \mathrm{mmHg}$ & $30 \mathrm{mmHg}$ \\
\hline 10 & $212(179-277)$ & $286(259-337)$ & $329(316-345)$ \\
\hline 9 & $228(202-295)$ & $306(275-346)$ & $337(322-357)$ \\
\hline 8 & $236(234-307)$ & $311(293-346)$ & $348(336-362)$ \\
\hline 7 & $260(223-288)$ & $292(286-348)$ & $356(330-384)$ \\
\hline 6 & $268(231-315)$ & $340(279-497)$ & $342(317-419)$ \\
\hline 5 & $292(254-347)$ & $334(302-421)$ & $350(320-425)$ \\
\hline 4 & $329(253-371)^{\dagger}$ & $326(291-402)$ & $346(333-471)$ \\
\hline 3 & $307(256-386)$ & $325(299-398)$ & $349(341-511)$ \\
\hline 2 & $250(210-367)$ & $293(268-404)$ & $341(322-504)$ \\
\hline 1 & $111(82-165)^{*}$ & $256(184-400)$ & $330(299-502)$ \\
\hline 0 & $63(20-121)^{*}$ & $200(92-353)$ & $315(220-453)$ \\
\hline
\end{tabular}

Note:

P $<0.05$ vs. other positions;

${ }^{\dagger} \mathrm{P}<0.05 \mathrm{vs} .10 \mathrm{~cm}$ proximal to the midline of EGJ (position 0). 
Table 2

Median ( $5^{\text {th }}$ to $95^{\text {th }}$ percentile) distensibility index (DI, $\mathrm{mm}^{2} / \mathrm{mmHg}$ ) values for EGJ, esophageal body $2-5 \mathrm{~cm}$ proximal to the EGJ and 6-10 cm proximal to the EGJ during distension volume at 30, 40, 50 and $60 \mathrm{ml}$.

\begin{tabular}{|l|l|l|l|}
\hline Distension volume $(\mathbf{m l})$ & EGJ & $\mathbf{2 - 5} \mathbf{~ c m}$ proximal & $\mathbf{6 - 1 0} \mathbf{~ c m}$ proximal \\
\hline 30 & $3.2(1.0-11.6)^{*}$ & $22.0(6.3-25.0)$ & $11.0(5.0-26.0)$ \\
\hline 40 & $5.7(1.4-15.8)^{*}$ & $17.0(8.3-21.5)$ & $13.0(7.3-21.0)$ \\
\hline 50 & $5.9(1.6-9.3)^{*}$ & $14.2(7.9-23.4)$ & $10.0(6.7-14.0)$ \\
\hline 60 & $6.3(2.1-9.5)$ & $8.1(5.3-20.0)$ & $7.0(5.0-13.0)$ \\
\hline P values (Kruskal-Wallis) & 0.34 & 0.02 & 0.08 \\
\hline
\end{tabular}

Note:

P $<0.05$ vs. $2-5 \mathrm{~cm}$ proximal to the EGJ and 6-10 cm proximal to the EGJ. 